\title{
INDUCTIVE APPROACH TO EFFECTIVE B-SEMIAMPLENESS
}

\author{
ENRICA FLORIS
}

\begin{abstract}
An lc-trivial fibration is the data of a pair $(X, B)$ and a fibration such that $\left.\left(K_{X}+B\right)\right|_{F}$ is torsion where $F$ is the general fibre. For such a fibration we have an equality $K_{X}+B+1 / r(\varphi)=f^{*}\left(K_{Z}+B_{Z}+M_{Z}\right)$ where $B_{Z}$ is the discriminant of $f, M_{Z}$ is the moduli part and $\varphi$ is a rational function. It has been conjectured by Prokhorov and Shokurov that there exists an integer $m=m(r, \operatorname{dim} F)$ such that $m M_{Z^{\prime}}$ is base-point-free on some birational model $Z^{\prime}$ of $Z$. In this work we reduce this conjecture to the case where the base $Z$ has dimension one. Moreover in the case where $M_{Z} \equiv 0$ we prove the existence of an integer $m$, that depends only on the middle Betti number of a canonical covering of the fibre, such that $m M_{Z} \sim 0$.
\end{abstract}

\section{INTRODUCTION}

The canonical bundle formula is a tool for studying the properties of the canonical bundle of a variety $X$, such that there exists a fibration $f: X \rightarrow Z$, in terms of the properties of the canonical bundle of $Z$, of the singularities of the fibration and of the birational variation of the fibres of $f$.

More precisely we consider a pair $(X, B)$ such that there exists an lc-trivial fibration $f:(X, B) \rightarrow Z$, that is a fibration such that $\left.\left(K_{X}+B\right)\right|_{F}$ is a torsion divisor, where $F$ is a general fibre (see Section 2 for a complete definition). Then we can write

$$
K_{X}+B+\frac{1}{r}(\varphi)=f^{*}\left(K_{Z}+B_{Z}+M_{Z}\right)
$$

where $\varphi$ is a rational function and $r$ is the minimum integer such that $\left.r\left(K_{X}+B\right)\right|_{F} \sim 0$. The divisor $B_{Z}$ is called the discriminant and it is defined in terms of some log-canonical thresholds with respect to the pair $(X, B)$. Precisely we have $B_{Z}=\sum\left(1-\gamma_{p}\right) p$ where

$$
\gamma_{p}=\sup \left\{t \in \mathbb{R} \mid\left(X, B+t f^{*}(p)\right) \text { is lc over } p\right\} .
$$

The divisor $M_{Z}$, called the moduli part, is a $\mathbb{Q}$-Cartier divisor and it is nef on some birational modification of $Z$ by [2, Theorem 0.2]. The moduli part should be related to the birational variation of the fibres of $f$.

This is true for instance in the case of elliptic fibrations. Indeed, if $f: X \rightarrow Z$ is an elliptic fibration, we have Kodaira's canonical bundle formula

$$
K_{X}=f^{*}\left(K_{Z}+B_{Z}+M_{Z}\right)
$$

Date: August 17, 2021. 
and

$$
12 M_{Z} \sim j^{*} \mathcal{O}_{\mathbb{P}^{1}}(1)
$$

where $j: Z \rightarrow \mathbb{P}^{1}=\mathcal{M}_{1}$ is the application induced by $f$ to the moduli space of elliptic curves. In particular $M_{Z}$ is semiample.

In [20] Prokhorov and Shokurov state the following conjecture (in our statement we specify the dimension of the base).

EbS(k) 1.1 (Effective b-Semiampleness, Conjecture 7.13.3, [20]). There exists an integer number $m=m(d, r)$ such that for any lc-trivial fibration $f:(X, B) \rightarrow Z$ with dimension of the generic fibre $F$ equal to $d$, dimension of $Z$ equal to $k$ and Cartier index of $\left(F,\left.B\right|_{F}\right)$ equal to $r$ there exists a birational morphism $\nu: Z^{\prime} \rightarrow Z$ such that $m M_{Z^{\prime}}$ is base point free.

The relevance of the above conjecture is well illustrated for instance by a result due to X. Jiang, who proved recently in [12] that Conjecture EbS 1.1 implies a uniformity statement for the Iitaka fibration of any variety of positive Kodaira dimension under the assumption that the fibres have a good minimal model.

Moreover Todorov and Todorov-Xu prove some unconditional uniformity results for the Iitaka fibration of varieties of Kodaira dimension at most 2 and Kodaira codimension 1 (Todorov [22, Theorem 1.2]) and 2 (Todorov and $\mathrm{Xu}$ [23, Theorem 1.2]). Their proofs relie on the existence of a bound on the denominators of the moduli part and, for the case of Kodaira codimension one, on the result by Prokhorov and Shokurov that proved conjecture EbS for all $k$ in the case where the fibres are curves (see [20, Theorem 8.1]).

The semiampleness of the moduli part has been also proved for all $k$ if $F$ is isomorphic to a K3 surface or to an abelian variety by Fujino in [8, Theorem 1.2].

It is worth noticing that the proofs of semiampleness in these cases use the existence of a moduli space for the fibres.

The main goal of this work is to develop an inductive approach to the conjecture EbS. Our first result is the following.

\section{Theorem 1.2. $\boldsymbol{E b S}(1)$ implies $\boldsymbol{E} \boldsymbol{b} \boldsymbol{S}(\boldsymbol{k})$.}

An inductive approach on the dimension of the base as in Theorem 1.2 allows us to prove a result of effective semiampleness in the case $M_{Z} \equiv 0$. Indeed we are able to prove an effective version of [3, Theorem 3.5].

Theorem 1.3. There exists an integer number $m=m(b)$ such that for any klt-trivial fibration $f:(X, B) \rightarrow Z$ with

- $M_{Z} \equiv 0$;

- $\operatorname{Betti}_{\operatorname{dim} E^{\prime}}\left(E^{\prime}\right)=b$ where $E^{\prime}$ is a non-singular model of the cover $E \rightarrow F$ associated to the unique element of $\left|r\left(K_{F}+\left.B\right|_{F}\right)\right|$;

we have $m M_{Z} \sim \mathcal{O}_{Z}$.

Moreover for the case where the pair $(X, B)$ is lc but not klt on the generic point of the base we have the following 
INDUCTIVE APPROACH TO EFFECTIVE B-SEMIAMPLENESS

Theorem 1.4. Let $f:(X, B) \rightarrow Z$ be an lc-trivial fibration with $M_{Z} \equiv 0$. Then $M_{Z}$ is torsion.

In section 2 we recall some general definitions and results concerning the canonical bundle formula. Section 3 is devoted to the proof of Theorem 1.2. Section 4 and section 5 contain several results that will be useful in the proofs of Theorem 1.3 and Theorem 1.4 that are the subject of section 6 .

The techniques that we use in the proof of Theorem 1.3 come from the theory of variations of Hodge structures as in [3, Theorem 3.5]. The integer $m(b)$ is determined in the unipotent case by using the semisimplicity theorem of Deligne [6, Theorem 4.2.6]. Then we show that the same integer works in the general case.

The proof of Theorem 1.4 consists in adaptating the proof of Theorem 1.3 to the more general setting of variation of mixed Hodge structures.

Acknowledgements. I would like to express my gratitude to my Ph.D advisor, Gianluca Pacienza, for bringing my attention to this problem and for his generous help. This work owes a great deal to his influence.

\section{Notations, Definitions And KNOWn RESUlts}

We will work over $\mathbb{C}$. In the following $\equiv, \sim$ and $\sim_{\mathbb{Q}}$ will respectively indicate numerical, linear and $\mathbb{Q}$-linear equivalence of divisors. The following definitions are taken from [17].

Definition 2.1. A pair $(X, B)$ is the data of a normal variety $X$ and a $\mathbb{Q}$-Weil divisor $B$ such that $K_{X}+B$ is $\mathbb{Q}$-Cartier.

Definition 2.2. Let $(X, B)$ be a pair and write $B=\sum b_{i} B_{i}$. Let $\nu: Y \rightarrow X$ be a birational morphism, $Y$ normal. We can write

$$
K_{Y} \equiv \nu^{*}\left(K_{X}+B\right)+\sum a\left(E_{i}, X, B\right) E_{i}
$$

where $E_{i} \subseteq Y$ are distinct prime divisors and $a\left(E_{i}, X, B\right) \in \mathbb{R}$. Furthermore we adopt the convention that a nonexceptional divisor $E$ appears in the sum if and only if $E=\nu_{*}^{-1} B_{i}$ for some $i$ and then with coefficient $a(E, X, B)=-b_{i}$.

The $a\left(E_{i}, X, B\right)$ are called discrepancies.

$A$ divisor $E$ is exceptional over $X$ if there exists a birational morphism $\nu: Y \rightarrow X$ and $E \subseteq Y$ and it is exceptional for $\nu$.

Definition 2.3. Let $(X, B)$ be a pair and $f: X \rightarrow Z$ be a morphism. Let $o \in Z$ be a point. $A$ $\log$ resolution of $(X, B)$ over o is a birational morphism $\nu: X^{\prime} \rightarrow X$ such that for all $x \in f^{-1} O$ the divisor $\nu^{*}\left(K_{X}+B\right)$ is simple normal crossing at $x$.

Definition 2.4. We set

$$
\operatorname{discrep}(\mathrm{X}, \mathrm{B})=\inf \{\mathrm{a}(\mathrm{E}, \mathrm{X}, \mathrm{B}) \mid \mathrm{E} \text { exceptional divisor over } \mathrm{X}\} \text {. }
$$

A pair $(X, B)$ is defined to be 
- klt (kawamata log terminal) if $\operatorname{discrep}(\mathrm{X}, \mathrm{B})>-1$,

- lc (log canonical) if $\operatorname{discrep}(\mathrm{X}, \mathrm{B}) \geq-1$.

Definition 2.5. Let $f:(X, B) \rightarrow Z$ be a morphism and $o \in Z$ a point. For an exceptional divisor $E$ over $X$ we set $c(E)$ its image in $X$. We set

$\operatorname{discrep}_{\mathrm{o}}(\mathrm{X}, \mathrm{B})=\inf \{\mathrm{a}(\mathrm{E}, \mathrm{X}, \mathrm{B}) \mid$ E exceptional divisor over $\mathrm{X}, \mathrm{f}(\mathrm{c}(\mathrm{E}))=\mathrm{o}\}$.

A pair $(X, B)$ is defined to be

- klt over o (kawamata log terminal) if $\operatorname{discrep}_{\mathrm{o}}(\mathrm{X}, \mathrm{B})>-1$,

- lc over o (log canonical) if $\operatorname{discrep}_{\mathrm{o}}(\mathrm{X}, \mathrm{B}) \geq-1$.

Definition 2.6. Let $(X, B)$ be a pair. A place for $(X, B)$ is a prime divisor on some birational model $\nu: Y \rightarrow X$ of $X$ such that $a(E, X, B)=-1$. The image of $E$ in $X$ is called a centre.

Definition 2.7. Let $(X, B)$ be a pair and $\nu: X^{\prime} \rightarrow X$ a log resolution of the pair. We set

$$
A(X, B)=K_{X^{\prime}}-\nu^{*}\left(K_{X}+B\right)
$$

and

$$
A^{*}(X, B)=A(X, B)+\sum_{a(E, X, B)=1} E .
$$

Definition 2.8. A klt-trivial (resp. lc-trivial) fibration $f:(X, B) \rightarrow Z$ consists of a contraction of normal varieties $f: X \rightarrow Z$ and of a $\log$ pair $(X, B)$ satisfying the following properties:

(1) $(X, B)$ has klt (resp. lc) singularities over the generic point of $Z$;

(2) $\operatorname{rank} f_{*}^{\prime} \mathcal{O}_{X}(\lceil A(X, B)\rceil)=1$ (resp. $\left.\operatorname{rank} f_{*}^{\prime} \mathcal{O}_{X}\left(\left\lceil A^{*}(X, B)\right\rceil\right)=1\right)$ where $f^{\prime}=f \circ \nu$ and $\nu$ is a given log resolution of the pair $(X, B)$;

(3) there exists a positive integer $r$, a rational function $\varphi \in k(X)$ and a $\mathbb{Q}$-Cartier divisor $D$ on $Z$ such that

$$
K_{X}+B+\frac{1}{r}(\varphi)=f^{*} D
$$

Remark 2.9. Condition (2) is verified for instance if $B$ is effective because

$$
\left\lceil A^{*}(X, B)\right\rceil=\left\lceil K_{X^{\prime}}-\nu^{*}\left(K_{X}+B\right)+\sum_{a(E, X, B)=1} E\right\rceil
$$

is exceptional.

Remark 2.10. The smallest possible $r$ is the minimum of the set

$$
\left\{m \in \mathbb{N}\left|m\left(K_{X}+B\right)\right|_{F} \sim 0\right\}
$$

that is the Cartier index of the fibre. We will always assume that the $r$ that appears in the formula is the smallest one.

Definition 2.11. Let $p \subseteq Z$ be a codimension one point. The log canonical threshold of $f^{*}(p)$ with respect to the pair $(X, B)$ is

$$
\gamma_{p}=\sup \left\{t \in \mathbb{R} \mid\left(X, B+t f^{*}(p)\right) \text { is lc over } p\right\} .
$$


We define the discriminant of $f:(X, B) \rightarrow Z$ as

$$
B_{Z}=\sum_{p}\left(1-\gamma_{p}\right) p
$$

We remark that, since the above sum is finite, $B_{Z}$ is a $\mathbb{Q}$-Weil divisor.

Definition 2.12. Fix $\varphi \in \mathbb{C}(X)$ such that $K_{X}+B+\frac{1}{r}(\varphi)=f^{*} D$. Then there exists a unique divisor $M_{Z}$ such that we have

$$
K_{X}+B+\frac{1}{r}(\varphi)=f^{*}\left(K_{Z}+B_{Z}+M_{Z}\right)
$$

where $B_{Z}$ is as in (2.1). The $\mathbb{Q}$-Weil divisor $M_{Z}$ is called the moduli part.

We have the two following results.

Theorem 2.13 (Theorem 0.2 [2], [4]). Let $f:(X, B) \rightarrow Z$ be an lc-trivial fibration. Then there exists a proper birational morphism $Z^{\prime} \rightarrow Z$ with the following properties:

(i): $K_{Z^{\prime}}+B_{Z^{\prime}}$ is a $\mathbb{Q}$-Cartier divisor, and $\nu^{*}\left(K_{Z^{\prime}}+B_{Z^{\prime}}\right)=K_{Z^{\prime \prime}}+B_{Z^{\prime \prime}}$ for every proper birational morphism $\nu: Z^{\prime \prime} \rightarrow Z^{\prime}$.

(ii): $M_{Z^{\prime}}$ is a nef $\mathbb{Q}$-Cartier divisor and $\nu^{*}\left(M_{Z^{\prime}}\right)=M_{Z^{\prime \prime}}$ for every proper birational morphism $\nu: Z^{\prime \prime} \rightarrow Z^{\prime}$.

Proposition 2.14 (Proposition $5.5[2]$ ). Let $f:(X, B) \rightarrow Z$ be an lc-trivial fibration. Let $\tau: Z^{\prime} \rightarrow Z$ be a generically finite projective morphism from a non-singular variety $Z^{\prime}$. Assume there exists a simple normal crossing divisor $\Sigma_{Z^{\prime}}$ on $Z^{\prime}$ which contains $\tau^{-1} \Sigma_{Z}$ and the locus where $\tau$ is not étale. Let $M_{Z^{\prime}}$ be the moduli part of the induced lc-trivial fibration $f^{\prime}:\left(X^{\prime}, B^{\prime}\right) \rightarrow Z^{\prime}$. Then $M_{Z^{\prime}}=\tau^{*} M_{Z}$.

Theorem 2.15 (Inverse of adjunction, Proposition 3.4, [1], see also Theorem 4.5 [10]). Let $f:(X, B) \rightarrow Z$ be an lc-trivial fibration. Then $\left(Z, B_{Z}\right)$ has klt $(l c)$ singularities in a neighborhood of a point $p \in Z$ if and only if $(X, B)$ has klt (lc) singularities in a neighborhood of $f^{-1} p$.

The Formula (2.2) is called canonical bundle formula.

\section{REDUCTION THEOREMS}

Throughout this part we will assume that the bases of the lc-trivial fibrations are smooth varieties.

Lemma 3.1. Let $f:(X, B) \rightarrow Z$ be an lc-trivial fibration. Then there exists a hyperplane section $H \subseteq Z$ such that $\left.M_{Z}\right|_{H}=M_{H}$.

Proof. The set

$$
\mathcal{S}=\left\{\begin{array}{l}
o \text { point of } Z \text { of codimension } 1 \text { such that the log canonical } \\
\text { threshold of } f^{*} o \text { with respect to }(X, B) \text { is different from } 1
\end{array}\right\}
$$


is a finite set.

By the Bertini theorem, since $Z$ is smooth, we can find a hyperplane section $H \subseteq Z$ such that

(1) $H$ is smooth;

(2) $H$ intersects $o$ transversally and generically for every $o \in \mathcal{S}$;

(3) $H$ does not contain any intersection $o \cap o^{\prime}$ where $o^{\prime} \in \mathcal{S} \backslash\{o\}$.

The restriction $f_{H}: X \cap f^{-1}(H) \rightarrow H$ is again an lc-trivial fibration. Set

$$
X_{H}=f^{-1}(H) ; \quad B_{X_{H}}=\left.B\right|_{X_{H}} ; \quad o_{H}=o \cap H .
$$

The canonical bundle formula for $f_{H}$ is

$$
K_{X_{H}}+B_{X_{H}}+\frac{1}{r}(\psi)=f_{H}^{*}\left(K_{H}+B_{H}+M_{H}\right) .
$$

Then the log canonical threshold of $f_{H}^{*} o_{H}$ with respect to $\left(X_{H}, B_{H}\right)$ is equal to the log canonical threshold of $f^{*} O$ with respect to $(X, B)$. We have then $\left.B_{Z}\right|_{H}=B_{H}$.

If we write the canonical bundle formula for $f$, we have

$$
K_{X}+B+\frac{1}{r}(\varphi)=f^{*}\left(K_{Z}+B_{Z}+M_{Z}\right)
$$

If we sum $f^{*} H$ on both sides of the equality, restrict to $f^{-1} H=X_{H}$ and apply the adjunction formula, we obtain

$$
K_{X_{H}}+B_{X_{H}}+\frac{1}{r}\left(\left.\varphi\right|_{X_{H}}\right)=f_{H}^{*}\left(K_{H}+\left.B_{Z}\right|_{H}+\left.M_{Z}\right|_{H}\right) .
$$

Since we have $\left.B_{Z}\right|_{H}=B_{H}$, we must also have $\left.M_{Z}\right|_{H}=M_{H}$.

Lemma 3.1 is the main tool in order to prove by induction Theorem 1.2 .

Proposition 3.2. Conjecture $\boldsymbol{E b S ( 1 )}$ implies that for all lc-trivial fibration $f: X \rightarrow Z$ we have

$$
\operatorname{codim}\left(\operatorname{Bs}\left|m M_{Z}\right|\right) \geq 2
$$

where $m$ is as in Conjecture $\boldsymbol{E b S ( 1 ) . ~}$

Proof. We prove the statement by induction on $k=\operatorname{dim} Z$. The base of induction is $\operatorname{dim} Z=1$ and this case follows from $\mathbf{E b S ( 1 ) . ~ S u p p o s e ~ t h e n ~ t h a t ~ t h e ~ s t a t e m e n t ~ i s ~ t r u e ~ f o r ~ l c - t r i v i a l ~}$ fibration whose base hase dimension $k-1$ and let $f: X \rightarrow Z$ be an lc-trivial fibration with $\operatorname{dim} Z=k>1$. Then we have

$$
\left|m M_{Z}\right|=|M|+\text { Fix }
$$

where Fix is the fixed part of the linear system and $\operatorname{codim}(\operatorname{Bs}|M|) \geq 2$. Let $H$ be a hyperplane section as in Lemma 3.1, such that $H-m M_{Z}$ is ample. By the Kodaira vanishing theorem

$$
h^{0}\left(Z, m M_{Z}-H\right)=h^{1}\left(Z, m M_{Z}-H\right)=0
$$

and the restriction induces an isomorphism

$$
H^{0}\left(Z, m M_{Z}\right) \cong H^{0}\left(H,\left.m M_{Z}\right|_{H}\right) \cong H^{0}\left(H, m M_{H}\right)
$$


Then if we write

$$
\begin{aligned}
\left|m M_{Z}\right|_{\mid H} & =|M|_{\mid H}+\left.\mathrm{Fix}\right|_{H} \\
\left|m M_{H}\right| & =|L|+\mathrm{fix}
\end{aligned}
$$

where fix is the fixed component of the linear system $\left|m M_{H}\right|$, we have fix $\supseteq$ Fix $\left.\right|_{H}$. And since by inductive hypothesis fix $=0$ also $\left.\operatorname{Fix}\right|_{H}=0$ and then Fix $=0$.

Corollary 3.3. Conjecture $\boldsymbol{E} \boldsymbol{b} \boldsymbol{S}(\mathbf{1})$ implies that for any lc-trivial fibration $f: X \rightarrow Z$ we have $h^{0}\left(Z, m M_{Z}\right) \geq 2$, unless $M_{Z}$ is torsion, where $m$ is as in $\boldsymbol{E b S ( 1 )}$.

Proof. By Proposition 3.2 there must be at least two sections, unless $M_{Z}$ is torsion.

Proof of Theorem 1.2. We treat first the torsion case, we prove by induction on the dimension of the base of the lc-trivial fibration that there exists an integer $m=m(d, r)$ such that $m M_{Z} \cong \mathcal{O}_{Z}$. If the dimension of the base equals one then it follows from Conjecture $\mathbf{E b S}(\mathbf{1})$. Assume then that $f: X \rightarrow Z$ is an lc-trivial fibration with $\operatorname{dim} Z=k>1$ and $M_{Z}$ is torsion, that is there exists an integer $a$ such that $a M_{Z} \cong \mathcal{O}_{Z}$.

Let $H$ be a hyperplane section such that $\left.M_{Z}\right|_{H}=M_{H}$, as in Lemma 3.1, and such that $H-m M_{Z}$ is an ample divisor. Since $\left.M_{Z}\right|_{H}=M_{H}$, also $M_{H}$ is torsion because

$$
\left.\left.\mathcal{O}_{H} \cong \mathcal{O}_{Z}\right|_{H} \cong a M_{Z}\right|_{H} \cong a M_{H}
$$

By the Kodaira vanishing theorem, since $k>1$ and $H-m M_{Z}$ is an ample divisor, we have

$$
H^{0}\left(Z, m M_{Z}\right) \cong H^{0}\left(H, m M_{H}\right) .
$$

By the inductive hypothesis $m M_{H}$ is base-point-free, then $h^{0}\left(H, m M_{H}\right)=1$. Thus also $h^{0}\left(Z, m M_{Z}\right)=1$ and $m M_{Z} \cong \mathcal{O}_{Z}$.

Then we assume that $M_{Z}$ is not torsion and we prove the statement by induction on the dimension of the base of the lc-trivial fibration. The one-dimensional case is exactly Conjecture $\mathbf{E b S}(\mathbf{1})$. Suppose then that the statement is true for all the lc-trivial fibrations whose base has dimension $k-1$ and let $f: X \rightarrow Z$ be an lc-trivial fibration with $\operatorname{dim} Z=k$. Let $Z^{\prime} \rightarrow Z$ be the birational model given by Theorem 2.13 (ii). We prove that $m M_{Z^{\prime}}$ is base-point-free. Let $\nu: \hat{Z} \rightarrow Z^{\prime}$ be a resolution of the linear system $\left|m M_{Z^{\prime}}\right|$. Then $\nu^{*}\left|m M_{Z^{\prime}}\right|=\mid$ Mob $\mid+$ Fix where $|\mathrm{Mob}|$ is a base-point-free linear system and Fix is the fixed part. We have

$$
\nu^{*}\left|m M_{Z^{\prime}}\right|=\left|\nu^{*}\left(m M_{Z^{\prime}}\right)\right|=\left|m M_{\hat{Z}}\right| .
$$

Since by Proposition 3.2 we have $\operatorname{codim}\left(\operatorname{Bs}\left|m M_{\hat{Z}}\right|\right) \geq 2$, it follows that Fix $=0$ and $\left|m M_{Z^{\prime}}\right|$ is base-point-free.

Remark 3.4. By considering as in Proposition 3.2 the long exact sequence associed to

$$
0 \rightarrow \mathcal{O}_{Z}\left(m M_{Z}-H\right) \rightarrow \mathcal{O}_{Z}\left(m M_{Z}\right) \rightarrow \mathcal{O}_{H}\left(\left.m M_{Z}\right|_{H}\right) \rightarrow 0
$$

for a hyperplane section $H$ as in Lemma 3.1, it is possible to also prove an inductive result on effective non-vanishing. That is, the existence of an integer $m=m(d, r)$ such that $H^{0}\left(Z, m M_{Z}\right) \neq 0$ for all lc-trivial fibrations $f:(X, B) \rightarrow Z$ with $\operatorname{dim} Z=1$ implies the same result for lc-fibrations with $\operatorname{dim} Z=k \geq 1$ (and with same dimension of the fibres and Cartier index). 


\section{VARIATION OF HODGE STRUCTURES AND COVERING TRICKS}

4.1. Variation of Hodge structures. Let $\mathcal{S}$ be $\mathbb{C}^{*}$ viewed as an $\mathbb{R}$-algebra.

Definition 4.1 (2.1.4 [6]). A real Hodge structure is a real vector space $V$ of finite dimension together with an action of $\mathcal{S}$.

The representation of $\mathcal{S}$ on $V$ induces a bigraduation on $V$, such that $\overline{V^{p q}}=V^{q p}$. We say that $V$ has weight $n$ if $V^{p q}=0$ whenever $p+q \neq n$.

Definition 4.2 (2.1.10 [6]). A Hodge structure $H$ of weight $n$ is

- a $\mathbb{Z}$-module of finite type $H_{\mathbb{Z}}$;

- a real Hodge structure of weight $n$ on $H_{\mathbb{R}}=H_{\mathbb{Z}} \otimes_{\mathbb{Z}} \mathbb{R}$.

Definition 4.3. Let $S$ be a topological space. A local system on $S$ is a sheaf $\mathbb{V}$ of $\mathbb{Q}$-vector spaces on $S$.

Let now $S$ be a complex manifold.

Definition 4.4. Let $\mathcal{V} \rightarrow S$ be a vector bundle. A connection is a morphism

$$
\nabla: \mathcal{V} \rightarrow \Omega_{S}^{1} \otimes \mathcal{V}
$$

that satisfies the Leibniz rule.

The curvature of a connection is $\nabla \circ \nabla: \mathcal{V} \rightarrow \Omega_{S}^{2} \otimes \mathcal{V}$. $A$ connection is said to be integrable if $\nabla \circ \nabla=0$.

By [5, Proposition 2.16] the data of a local system $\mathbb{V}$ is equivalent to the data of a vector bundle $\mathcal{V} \rightarrow S$ together with an integrable connection $\nabla$ and the correspondance is given by associating to $\mathbb{V}$ the vector bundle

$$
\mathcal{V}=\mathbb{V} \otimes \mathcal{O}
$$

Definition 4.5. A flat subsystem of a local system $\mathbb{V}$ is a sub-local system $\mathbb{W}$ of $\mathbb{V}$ or equivalently a subbundle $\mathcal{W}$ of $\mathcal{V}$ on which the curvature of the connection is zero.

Definition 4.6 ((3.1) [21]). A variation of Hodge structure of weight $m$ on $S$ is:

- a local system $\mathbb{V}$ on $S$;

- a flat bilinear form

$$
Q: \mathcal{V} \times \mathcal{V} \rightarrow \mathbb{C}
$$

which is rational with respect to $\mathbb{V}$, where $\mathcal{V}=\mathbb{V} \otimes \mathcal{O}_{S}$;

- a Hodge filtration $\left\{\mathcal{F}^{p}\right\}$, that is a decreasing filtration of $\mathcal{V}$ by holomorphic subbundles such that for all $p$ we have $\nabla\left(\mathcal{F}^{p}\right) \subseteq \Omega_{S}^{1} \otimes \mathcal{F}^{p-1}$.

Definition 4.7 ((3.4) [21]). A variation of mixed Hodge structure on $S$ is:

- a local system $\mathbb{V}$ on $S$;

- a Hodge filtration $\left\{\mathcal{F}^{p}\right\}$ that is a decreasing filtration of $\mathcal{V}$ by holomorphic subbundles such that for all $p$ we have $\nabla\left(\mathcal{F}^{p}\right) \subseteq \Omega_{S}^{1} \otimes \mathcal{F}^{p-1}$;

- a Weight filtration $\left\{\mathcal{W}_{k}\right\}$ that is an increasing filtration of $\mathcal{V}$ by local subsystems, or equivalently, the subsheaf $\mathcal{W}_{k}$ is defined over $\mathbb{Q}$ for every $k$; 
Moreover we require that the filtration induced by $\left\{\mathcal{F}^{p}\right\}$ on $\mathcal{W}_{k} / \mathcal{W}_{k-1}$ determines a variation of Hodge structure of weight $k$.

From now on we will be interested in variations of Hodge structures and of mixed Hodge structures defined on a Zariski open subset $Z_{0}$ of a projective variety $Z$. We assume moreover that $\Sigma_{Z}=Z \backslash Z_{0}$ is a simple normal crossing divisor.

The following is a fundamental result about the behaviour of a variation of Hodge structures on $Z_{0}$ near $\Sigma_{Z}$. For the definition of monodromy and unipotent monodromy of variations of Hodge structures and residue of a connection see [19, Definition 10.16, section 11.1.1]

Proposition 4.8 (Proposition 5.2(d), [5]). Let $\mathcal{V}$ be a variation of Hodge structures on $Z_{0}$ that has unipotent monodromies around $\Sigma_{Z}$. Let $z$ be a local variable with centre in $\Sigma_{Z}$. Then

a: There exists a unique extension $\tilde{\mathcal{V}}$ of $\mathcal{V}$ on $Z$ such that

$\mathrm{i}$ : every horizontal section of $\mathcal{V}$ as a section of $\tilde{\mathcal{V}}$ on $Z_{0}$ grows at most as

$$
O\left(\log \|z\|^{k}\right)
$$

near $\Sigma_{Z}$

ii: let $\mathcal{V}^{*}$ be the dual of $\mathcal{V}$. Every horizontal section of $\mathcal{V}^{*}$ grows at most as

$$
O\left(\log \|z\|^{k}\right)
$$

near $\Sigma_{Z}$

b: Conditions (i) and (ii) are equivalent respectively to conditions (iii) and (iv).

iii: The matrix of the connection on $\mathcal{V}$ on a local frame for $\tilde{\mathcal{V}}$ has logarithmic poles near $\Sigma_{Z}$.

iv: Each residue of the connection along each irreducible component of $\Sigma_{Z}$ is nilpotent.

c: Let $\mathcal{V}_{1}$ and $\mathcal{V}_{2}$ be variations of Hodge structures on $Z_{0}$ that has unipotent monodromies around $\Sigma_{Z}$. Every morphism $f: \mathcal{V}_{1} \rightarrow \mathcal{V}_{2}$ extends to a morphism $\tilde{\mathcal{V}}_{1} \rightarrow \tilde{\mathcal{V}}_{2}$. Moreover the functor $\mathcal{V} \mapsto \tilde{\mathcal{V}}$ is exact and commutes with $\otimes, \wedge$, Hom.

The extension $\tilde{\mathcal{V}}$ is called canonical extension.

Remark 4.9. In the situation of Proposition 4.8 the matrix of the connection on $\mathcal{V}$ has the following form

$$
\Gamma=\sum U_{i} \frac{d z_{i}}{z_{i}}
$$

where $U_{i}$ is the matrix that represents the nilpotent part of the monodromy around the component $\Sigma_{i}$ of $\Sigma_{Z}$.

Let $V, Z$ be nonsingular projective varieties and $h: V \rightarrow Z$ a surjective morphism with connected fibres. Let $Z_{0} \subseteq Z$ be the largest Zariski open set where $h$ is smooth and $V_{0}=$ $h^{-1} Z_{0}$. Assume that $\Sigma_{Z}=Z \backslash Z_{0}$ and $\Sigma_{V}=V \backslash V_{0}$ are simple normal crossing divisors on $Z$ and $V$. Set $d=\operatorname{dim} V-\operatorname{dim} Z$.

Consider $\mathcal{H}_{\mathbb{C}}=\left(R^{d} h_{*} \mathbb{C}_{V_{0}}\right)_{\text {prim }}$ and $\mathcal{H}_{0}=\mathcal{H}_{\mathbb{C}} \otimes \mathcal{O}_{Z_{0}}$ and set $\mathcal{F}=h_{*} \omega_{V / Z}$ and $\mathcal{F}_{0}=\mathcal{F} \otimes \mathcal{O}_{Z_{0}}$. We have that $\mathcal{H}_{\mathbb{C}}$ is a local system over $Z_{0}$. Moreover $\mathcal{H}_{0}$ has a descending filtration $\left\{\mathcal{F}^{p}\right\}_{0 \leq p \leq d}$, 
the Hodge filtration and $\mathcal{F}_{0}=\mathcal{F}^{d}$.

There is a canonical way to extend $\mathcal{H}_{0}$ and $\mathcal{F}_{0}$ on $Z$ :

Theorem 4.10 (Proposition 5.4 [5], Theorem 2.6 [16]). ical extension to a locally free sheaf on $Z$.

(1) $R^{d} h_{*} \mathbb{C}_{V_{0}} \otimes \mathcal{O}_{Z_{0}}$ has a canon-

(2) $h_{*} \omega_{V / Z}$ coincides with the canonical extension of the bottom piece of the Hodge filtration.

Let $h_{0}: V_{0} \rightarrow Z_{0}$ be as before. Let $D \subseteq V$ be a simple normal crossing divisor such that the restriction $\left.h_{0}\right|_{D}$ is flat. Assume that $D+\Sigma_{V}$ is simple normal crossing. Let us denote the restriction as

$$
h_{0}^{\prime}: V_{0} \backslash D \rightarrow Z_{0} .
$$

Thus $R^{d}\left(h_{0}^{\prime}\right)_{*} \mathbb{C}_{V_{0} \backslash D}$ is a local system on $Z_{0}$ by [21, section 5.2]. Let $\left\{\mathcal{F}^{p}\right\}$ be the Hodge filtration and let

$$
\mathcal{W}_{k}=\left(h_{0}\right)_{*} \Omega_{V_{0} / Z_{0}}^{k}(\log D) \otimes \Omega_{V_{0} / Z_{0}}^{\bullet-k}=\left\{\left(h_{0}\right)_{*} \Omega_{V_{0} / Z_{0}}^{k}(\log D) \otimes \Omega_{V_{0} / Z_{0}}^{s-k}\right\}_{s}
$$

be the weight filtration of the complex $\left(h_{0}\right)_{*} \Omega_{V_{0} / Z_{0}}^{\bullet}(\log D)$. In particular $\mathcal{W}_{k}$ is a complex. When we will need to refer to the trace left by $\mathcal{W}_{k}$ on an element $\left(h_{0}\right)_{*} \Omega_{V_{0} / Z_{0}}^{s}(\log D)$ of the complex we will write $\mathcal{W}_{k}\left(\left(h_{0}\right)_{*} \Omega_{V_{0} / Z_{0}}^{s}(\log D)\right)$.

Let $h: V \rightarrow Z$ be a morphism such that $\Sigma_{Z}$ is a simple normal crossing divisor and let $\tau: Z^{\prime} \rightarrow Z$ be a morphism from a nonsingular variety $Z^{\prime}$ such that $\tau^{-1} \Sigma_{Z}$ is a simple normal crossing divisor. Let $V^{\prime}$ be a desingularization of the component of $V \times{ }_{Z} Z^{\prime}$ that dominates $Z^{\prime}$.

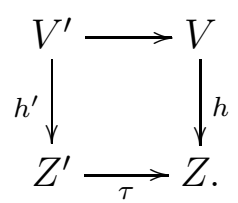

Assume now that $h^{\prime}$ and $h$ are such that $R^{d} h_{*} \mathbb{C}_{V_{0}}$ and $R^{d} h_{*}^{\prime} \mathbb{C}_{V_{0}^{\prime}}$ have unipotent monodromies. By Proposition 4.8[c] we have a commutative diagram of sheaves on $Z^{\prime}$

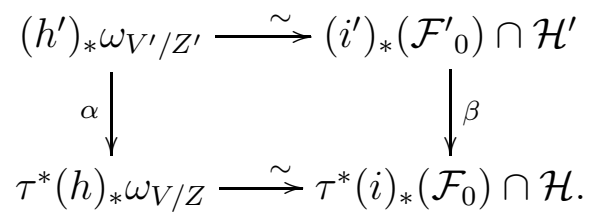

where $i: Z_{0} \rightarrow Z, i^{\prime}: Z_{0}^{\prime} \rightarrow Z^{\prime}$ are inclusions, $\mathcal{H}$ (resp. $\mathcal{H}^{\prime}$ ) is the canonical extension of $R^{d} h_{*} \mathbb{C}_{V_{0}}$ (resp. $\left.R^{d} h_{*}^{\prime} \mathbb{C}_{V_{0}^{\prime}}\right), \mathcal{F}_{0}=h_{*} \omega_{V /\left.Z\right|_{Z_{0}}}$ (resp. $\mathcal{F}^{\prime}{ }_{0}=h_{*}^{\prime} \omega_{V^{\prime} /\left.Z^{\prime}\right|_{Z_{0}^{\prime}}}$ ) and $\alpha, \beta$ are the pullbacks by $\tau$. If we have an isomorphism

$$
\alpha:\left(h^{\prime}\right)_{*} \omega_{V^{\prime} / Z^{\prime}} \rightarrow \tau^{*}(h)_{*} \omega_{V / Z}
$$

then for all $p \in Z^{\prime}$ we have an isomorphism of $\mathbb{C}$-vector spaces

$$
\alpha_{p}:\left(\left(h^{\prime}\right)_{*} \omega_{V^{\prime} / Z^{\prime}}\right)_{p} \rightarrow\left(h_{*} \omega_{V / Z}\right)_{\tau(p)} .
$$


If $\tau$ is a birational automorphism of $Z$ that fixes $p$, then $\alpha_{p}$ is an element of the linear group of $\left((h)_{*} \omega_{V / Z}\right)_{p}$. In particular we have the following:

Proposition 4.11. Let $h: V \rightarrow Z$ be a fibration such that $R^{d} h_{*} \mathbb{C}_{V_{0}}$ has unipotent monodromies. Assume that we have an action of a group $G$ on $Z$ given by a homomorphism

$$
G \rightarrow \operatorname{Bir}(Z)=\{\nu: Z \rightarrow Z \mid \nu \text { is birational }\} .
$$

Let $G_{p}$ be the stabilizer of $p \in Z$. Then we have an induced action of $G_{p}$ on $\mathcal{H}_{p}$ and on $\left(h_{*} \omega_{V / Z}\right)_{p}$ and these actions commute with the inclusion $\left(h_{*} \omega_{V / Z}\right)_{p} \subseteq \mathcal{H}_{p}$.

By [14, Theorem 17] we can assume that $R^{d} h_{*} \mathbb{C}_{V_{0}}$ (or, more in general, a local system that has quasi-unipotent monodromies) has unipotent monodromies modulo a finite base change by a Galois morphism. We restate Kawamata's result in a more precise way that is useful for our purposes.

Theorem 4.12 (Theorem 17, Corollary 18 [14]). Let $h: V \rightarrow Z$ be an algebraic fibre space. Let $Z_{0} \subseteq Z$ be the largest Zariski open set where $h$ is smooth and $V_{0}=h^{-1} Z_{0}$. Assume that $\Sigma_{Z}=Z \backslash Z_{0}$ and $\Sigma_{V}=V \backslash V_{0}$ are simple normal crossing divisors on $Z$ and $V$. Set $d=\operatorname{dim} V-\operatorname{dim} Z$.

Then there exists a finite surjective morphism $\tau: Z^{\prime} \rightarrow Z$ from a nonsingular projective algebraic variety $Z^{\prime}$ such that for a desingularization $V^{\prime}$ of $V \times{ }_{Z} Z^{\prime}$ the morphism $h^{\prime}: V^{\prime} \rightarrow Z^{\prime}$ induced from $h$ is such that $R^{d} h_{*}^{\prime} \mathbb{C}_{V_{0}^{\prime}}$ has unipotent monodromies.

Moreover $\tau$ is a composition of cyclic coverings $\tau_{j}$

$$
\tau: Z^{\prime}=Z_{k+1} \stackrel{\tau_{k}}{\rightarrow} Z_{k} \ldots Z_{2} \stackrel{\tau_{1}}{\rightarrow} Z_{1}=Z
$$

where $\tau_{j}$ is defined by the building data

$$
A_{j}^{\otimes \delta_{j}} \sim H_{j}
$$

where $A_{j}$ is very ample on $Z_{j}$ and $H_{j}$ is simple normal crossing.

We have the following results by Deligne. We state them in our situation, but they hold in a more general setting.

Theorem 4.13 (Theorem 4.2.6 [6]). The representation of the fundamental group $\pi_{1}\left(Z_{0}, z\right)$ on the fibre $\mathcal{H}_{\mathbb{Q}, z}$ is semi-simple.

Here we prove a slight modification of [6, Corollary 4.2.8(ii)(b)].

Corollary 4.14. Let $W$ be a local subsystem of $\mathcal{V}_{\mathbb{C}}$ of rank one. Let $b=\operatorname{dim} \mathcal{V}$. Let

$$
m(x)=\operatorname{lcm}\{k \mid \phi(k) \leq x\}
$$

where $\phi$ is the Euler function. Then $W^{\otimes m(b)}$ is a trivial local system.

Proof. By Theorem 4.13 we can write

$$
\mathcal{H}_{\mathbb{C}, z}=\bigoplus_{i=1}^{r} \mathcal{H}_{i}
$$


where the $\mathcal{H}_{i}$ are the isotypic compontents (that is the components that are direct sum of simple representations of the same weight). Since $\operatorname{dim} W=1$, the subspace $W_{z}$ is contained in one isotypic component, let's say $\mathcal{H}_{1}$. We have

$$
\mathcal{H}_{1}=\mathcal{H}_{\lambda}^{\oplus k}
$$

where $\mathcal{H}_{\lambda}$ is a simple component of weight $\lambda$. Since $W_{z}$ is simple, it identifies to one of the $\mathcal{H}_{\lambda}$ 's and then

$$
\bigwedge^{k} \mathcal{H}_{1} \cong W_{z}^{\otimes k}
$$

If $\chi$ is the character that determines $W_{z}$ as a representation then the character $\chi^{k}$ determines $\bigwedge^{k} \mathcal{H}_{1}$. Let $\mathcal{S}$ be the real algebraic group $\mathbb{C}^{*}$. We have an action of $\mathcal{S}$ on $\mathcal{H}_{1}$. Indeed by [6, Corollary 4.2.8(ii)(a)] for all $t \in \mathcal{S}$ we have $t \mathcal{H}_{1} \cong \mathcal{H}_{1}$. In particular $t \mathcal{H}_{1}$ and $\mathcal{H}_{1}$ have the same weight. But since $\mathcal{H}_{1}$ is isotypic, we have $t \mathcal{H}_{1}=\mathcal{H}_{1}$. The vector space $\hat{\mathcal{H}}_{1}=\mathcal{H}_{1}+\overline{\mathcal{H}}_{1}$ is real and $\mathcal{S}$-invariant, thus it is defined by a real Hodge substructure of $\mathcal{H}_{\mathbb{R}, z}$. Thus a polarization on $\mathcal{H}$ induces a non-degenerate bilinear form on $\hat{\mathcal{H}}_{1}$ that is invariant under the action of $\pi_{1}\left(Z_{0}, z\right)$.

Let $e=\operatorname{dim} \hat{\mathcal{H}}_{1}$. The Hodge structure on $\hat{\mathcal{H}}_{1}$ comes from an integer structure and thus $\left(\bigwedge^{e} \hat{\mathcal{H}}_{1}\right)^{\otimes 2}$ is trivial because $\pi_{1}\left(Z_{0}, z\right)$ can act on a $\mathbb{Z}$-module of rank one only by \pm 1 . Since $\hat{\mathcal{H}}_{1}=\mathcal{H}_{1}+\overline{\mathcal{H}}_{1}$, there are two possibilities:

(a): if $\mathcal{H}_{1}$ is real, the character $\chi^{2 k}$ is trivial

(b): else $\chi^{2 k} \bar{\chi}^{2 k}$ is trivial.

In any case $|\chi|=1$.

The representation of $\pi_{1}\left(Z_{0}, z\right)$ on $\mathcal{H}_{\mathbb{C}, z}$ comes from a representation on $\mathcal{H}_{\mathbb{Q}, z}$ and all the conjugated representations of $W_{z}$ appear in $\mathcal{H}_{\mathbb{C}, z}$. Thus we have at most $b=\operatorname{dim} \mathcal{H}$ conjugated representations of $W_{z}$.

We proved that for all $\left.\gamma \in \pi_{1} Z_{0}, z\right)$ the number $\chi(\gamma)$ is a complex number of module one and with at most $b$ complex conjugates. Thus $\chi(\gamma)$ is a $k$-th root of unity, with $k \leq b$. If we define

$$
m(b)=\operatorname{lcm}\{k \mid \phi(k) \leq b\}
$$

where $\phi$ is the Euler function, then $\chi^{m(b)}$ is trivial.

4.2. Covering tricks. In order to give an interpretation of the moduli part in terms of variation of Hodge structures we need to consider an auxiliary $\log$ pair $\left(V, B_{V}\right)$ with a fibration $h: V \rightarrow Z$.

Let $f: X \rightarrow Z$ be an lc-trivial fibration. Set $\Sigma_{Z}=\operatorname{Supp} B_{Z}$ and we assume that $\Sigma_{Z}$ is a simple normal crossing divisor. Set $\Sigma_{X}=\operatorname{Supp} f^{*} \Sigma_{Z}$ and assume that $B+\Sigma_{X}$ has simple normal crossing support. We define $g: V \rightarrow X$ as the desingularization of the covering induced by the field extension

$$
\mathbb{C}(X) \subseteq \mathbb{C}(X)(\sqrt[r]{\varphi})
$$


that is, the desingularization of the normalization of $X$ in $\mathbb{C}(X)(\sqrt[r]{\varphi})$ where $\varphi$ is as in (2.2). Let $B_{V}$ be the divisor defined by the equality $K_{V}+B_{V}=g^{*}\left(K_{X}+B\right)$. Set $h=f \circ g: V \rightarrow Z$. Then $h$ and $f$ induce the same discriminant and moduli divisor. Let $\Sigma_{V}$ be the support of $h^{*} \Sigma_{Z}$ and assume that $\Sigma_{V}+B_{V}$ has simple normal crossing support.

The Galois group of (4.1) is cyclic of order $r$, then we have an action of

$$
\mu_{r}=\left\{x \in \mathbb{C} \mid x^{r}=1\right\}
$$

on $g_{*} \mathcal{O}_{V}$. Then we have also an action of $\mu_{r}$ on $h_{*} \omega_{V / Z}$ and on $h_{*} \omega_{V / Z}\left(P_{V}\right)$ where $P_{V}$ are the horizontal places of the pair $\left(V, B_{V}\right)$.

Proposition 4.15 (Claim 8.4.5.5, Section 8.10.3 [4]). Let $f: X \rightarrow Z$ and $V \rightarrow X$ be as above. The decomposition in eigensheaves is

$$
h_{*} \omega_{V / Z}=\bigoplus_{i=0}^{r-1} f_{*} \mathcal{O}_{X}\left(\left\lceil(1-i) K_{X / Z}-i B+i f^{*} B_{Z}+i f^{*} M_{Z}\right\rceil\right) .
$$

Let $P_{V}$ be the places of $\left(V, B_{V}\right)$ and $P$ the places of $(X, B)$. Then we have

$$
h_{*} \omega_{V / Z}\left(P_{V}\right)=\bigoplus_{i=0}^{r-1} f_{*} \mathcal{O}_{X}\left(\left\lceil(1-i) K_{X / Z}-i B+P+i f^{*} B_{Z}+i f^{*} M_{Z}\right\rceil\right)
$$

and the righthand-side is the eigensheaf decomposition of the left-hand-side with respect to the action of $\mu_{r}$.

Proposition 4.16 (Proposition 5.2, [2]). Assume that $R^{d} h_{*} \mathbb{C}_{V_{0}}$ has unipotent monodromies. Then $M_{Z}$ is an integral divisor and it is equal to the eigensheaf $f_{*} \mathcal{O}_{X}\left(\left\lceil-B+P+f^{*} B_{Z}+f^{*} M_{Z}\right\rceil\right)$ corresponding to a fixed primitive rth root of unity.

\section{BOUNDing THE DENOMINATORS OF THE MODULI PART}

Conjecture EbS 1.1 implies in particular the existence of an integer $N=N(d, r)$ such that for all $f:(X, B) \rightarrow Z$ lc-trivial fibration with fibres of dimension $d$ and Cartier index of $\left(F,\left.B\right|_{F}\right)$ equal to $r$ the divisor $N M_{Z}$ has integer coefficients. The result was proved in [22, Theorem 3.2] when the fibre is a rational curve. In [7], by a different method, we found such integer which is considerably smaller than the one in [22]. For the reader convenience we present here an argument, due to Todorov [22, Theorem 3.2], valid in the general case.

Theorem 5.1. There exists an integer number $m=m(b)$ such that for any klt-trivial fibration $f:(X, B) \rightarrow Z$ with Betti $i_{\operatorname{dim} E^{\prime}}\left(E^{\prime}\right)=b$ where $E^{\prime}$ is a non-singular model of the cover of a general fibre of $f, E \rightarrow F$ associated to the unique element of $\left|r\left(K_{F}+\left.B\right|_{F}\right)\right|$ the divisor $m M_{Z}$ has integer coefficients.

We begin by reducing the problem to the case where the base $Z$ is a curve.

Proposition 5.2. If Theorem 5.1 holds for fibrations whose bases have dimension one then Theorem 5.1 holds for fibrations whose bases have dimension $k \geq 1$. 
Proof. We prove the statement by induction on $k=\operatorname{dim} Z$. If $k=1$ then it follows from the hypothesis. Assume the statement holds for fibrations over bases of dimension $k-1$ and we consider $f: X \rightarrow Z$ with $\operatorname{dim} Z=k$. Let $H$ be a hyperplane section of $Z$ as in Lemma 3.1. We have thus $\left.M_{Z}\right|_{H}=M_{H}$. Since $H$ is ample, it meets each component of $M_{Z}$ and we can choose it such that it meets transversally the components of $M_{Z}$. It follows that $N M_{Z}$ has integer coefficients if and only if $N M_{H}$ does, and we are done by inductive hypothesis.

Proof of Theorem 5.1. By Proposition 5.2 we can assume that $\operatorname{dim} Z=1$.

Consider a finite base change as in Theorem 4.12

$$
\tau: Z^{\prime} \rightarrow Z
$$

If $h^{\prime}: V^{\prime} \rightarrow Z^{\prime}$ is the induced morphism then $R^{d} h_{*}^{\prime} \mathbb{C}_{V_{0}^{\prime}}$ has unipotent monodromies. The covering $\tau$ is Galois and let $G$ be its Galois group. Then we have an action of $G$ on $Z^{\prime}$

$$
G \rightarrow \operatorname{Bir}\left(Z^{\prime}\right)=\operatorname{Aut}\left(Z^{\prime}\right)
$$

By abuse of notation we will denote as $g$ both an element of $G$ and its image in $\operatorname{Aut}\left(Z^{\prime}\right)$.

Let $p^{\prime} \in Z^{\prime}$ be a point and let $e$ be the ramification order of $\tau$ at $p^{\prime}$. Let $G_{p^{\prime}}$ be the stabilizer of $p^{\prime}$ with respect to the action (5.1). Set $\mu_{e}=\left\{x \in \mathbb{C} \mid x^{e}=1\right\}$.

There exists an analytic open set $p^{\prime} \in U \subseteq Z^{\prime}$ and a local coordinate $z$ on $U$ centered in $p^{\prime}$ such that for any $g \in G_{p^{\prime}}$ there exists $x \in \mu_{e}$ such that

$$
\begin{aligned}
\left.g\right|_{U}: \quad U & \longrightarrow U \\
z & \longmapsto x z .
\end{aligned}
$$

This induces a natural homomorphism

$$
G_{p^{\prime}} \rightarrow \mu_{e}
$$

Then the actions of $G_{p^{\prime}}$ given by Proposition 4.11 factorize through actions of $\mu_{e}$ :

$$
\begin{gathered}
\Phi: \mu_{e} \rightarrow G L\left(\left(R^{d} h_{*}^{\prime} \mathbb{C}_{V^{\prime}}\right)_{p^{\prime}}\right), \\
\Psi: \mu_{e} \rightarrow G L\left(\left(h_{*}^{\prime} \omega_{V^{\prime} / Z^{\prime}}\right)_{p^{\prime}}\right)
\end{gathered}
$$

that commute with the inclusion $\left(h_{*}^{\prime} \omega_{V^{\prime}} / Z^{\prime}\right)_{p^{\prime}} \subseteq\left(R^{d} h_{*}^{\prime} \mathbb{C}_{V^{\prime}}\right)_{p^{\prime}}$, that is such that for all $\zeta \in \mu_{e}$ the restriction of $\Phi(\zeta)$ to $\left(h_{*}^{\prime} \omega_{V^{\prime} / Z^{\prime}}\right)_{p^{\prime}}$ equals $\Psi(\zeta)$.

Thus on

$$
\left(h_{*}^{\prime} \omega_{V^{\prime} / Z^{\prime}}\right)_{p^{\prime}}=\bigoplus_{i=0}^{r-1} f_{*} \mathcal{O}_{X}\left(\left\lceil(1-i) K_{X / Z}-i B+i f^{*} B_{Z}+i f^{*} M_{Z}\right\rceil\right)
$$

we have two actions:

- one by the group $\mu_{e}$ that acts on $\varphi$ by a multiplication by an $e$-th rooth of unity,

- one by the group $\mu_{r}$ that acts on $\sqrt[r]{\varphi}$ by a multiplication by an $r$-th rooth of unity.

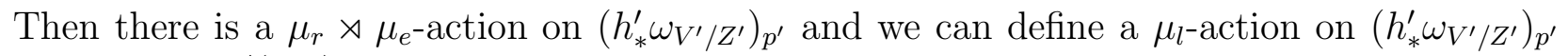
where $l=e r /(e, r)$. Since $\mu_{r} \subseteq \mu_{l}$ and this second group is commutative, the action of $\mu_{l}$ preserves the eigensheaves with respect to the action of $\mu_{r}$. By Proposition 4.16, the divisor 
$M_{Z^{\prime}}$ is an eigensheaf with respect to the action of $\mu_{r}$. Then $\mu_{l}$ acts on the stalk $\mathcal{O}_{Z^{\prime}}\left(M_{Z^{\prime}}\right) \otimes \mathbb{C}_{p^{\prime}}$ by a character $\chi_{p^{\prime}}$.

If for every $p^{\prime}$ and for every character $\chi_{p^{\prime}}$ the order of $\chi_{p^{\prime}}$ divides an integer $N$ then

$$
N M_{Z}=\left(\tau_{*} \mathcal{O}\left(N M_{Z^{\prime}}\right)\right)^{G}
$$

because by Proposition 2.14 we have $M_{Z^{\prime}}=\tau^{*} M_{Z}$. Thus $N M_{Z}$ is a Cartier divisor.

Let $\mathcal{H}^{\prime}$ be the canonical extension of the sheaf $\left(R^{d}\left(h_{0}^{\prime}\right)_{*} \mathbb{C}\right)_{\text {prim }} \otimes \mathcal{O}_{Z_{0}^{\prime}}$ to $Z^{\prime}$, where $d$ is the dimension of the fibre of $h^{\prime}$ and $h_{0}^{\prime}: V_{0}^{\prime} \rightarrow Z_{0}^{\prime}$ is the restriction to the smooth locus. The Hodge filtration also extends and its bottom piece is $h_{*}^{\prime} \mathcal{O}_{V^{\prime}}\left(K_{V^{\prime} / Z^{\prime}}\right)$. Then all the characters that are conjugated to $\chi_{p^{\prime}}$ must appear as subrepresentations of $\mathcal{H}_{p^{\prime}}^{\prime}$ (see also [․ Corollary 4.2.8(ii)(b)] or Corollary 4.14).

If $\chi_{p^{\prime}}$ acts by a primitive $k$-th root of unity, then its conjugated subrepresentations are $\phi(k)$ where $\phi$ is the Euler function. This bounds $k$ because then $\phi(l) \leq B_{d}$, where $B_{d}=h^{d}\left(E^{\prime}, \mathbb{C}\right)$ is the $d$-th Betti number.

Set $m(x)=\operatorname{lcm}\{k \mid \phi(k) \leq x\}$. Then $m\left(B_{d}\right) M_{Z}$ has integer coefficients.

\section{The CASE $M_{Z} \equiv 0$}

6.1. KLT-trivial fibrations with numerically trivial moduli part. The goal of this subsection is the proof of Theorem 1.3. As in Theorem 1.2 the problem can be reduced to the case where the base is a curve.

Proposition 6.1. Assume that there exists an integer number $m=m(b)$ such that for any klt-trivial fibration $f:(X, B) \rightarrow Z$ with

- $\operatorname{dim} Z=1$

- $M_{Z} \equiv 0$;

- Betti $i_{\operatorname{dim} E^{\prime}}\left(E^{\prime}\right)=b$ where $E^{\prime}$ is a non-singular model of the cover $E \rightarrow F$ associated to the unique element of $\left|r\left(K_{F}+\left.B\right|_{F}\right)\right|$;

we have $m M_{Z} \sim \mathcal{O}_{Z}$.

Then the same holds for bases $Z$ of arbitrary dimension.

Proof. We procede by induction on $k=\operatorname{dim} Z$. The base of induction is the hypothesis of the theorem.

Let us assume the statement holds for bases of dimension $k-1$ and prove it for a klt-fibration $f:(X, B) \rightarrow Z$ with $\operatorname{dim} Z=k$. Let $H$ be a hyperplane section, given by Lemma 3.1 , such that $\left.M_{Z}\right|_{H}=M_{H}$. Let $m$ be the integer given by the inductive hypothesis. Since $M_{Z} \equiv 0$ the divisor $H-m M_{Z}$ is ample. By taking the long exact sequence associated to

$$
0 \rightarrow \mathcal{O}_{Z}\left(m M_{Z}-H\right) \rightarrow \mathcal{O}_{Z}\left(m M_{Z}\right) \rightarrow \mathcal{O}_{H}\left(\left.m M_{Z}\right|_{H}\right) \rightarrow 0
$$

we obtain $H^{0}\left(Z, m M_{Z}\right) \cong H^{0}\left(H, m M_{H}\right)$ because $H^{i}\left(Z, m M_{Z}-H\right)=0$ for all $i<\operatorname{dim} Z$. Then $H^{0}\left(Z, m M_{Z}\right) \cong \mathbb{C}$, that implies $m M_{Z} \sim \mathcal{O}_{Z}$.

Proof of Theorem 1.3. By Proposition 6.1 applied in the case of a klt-trivial fibration, with $b=h^{d}\left(E^{\prime}, \mathbb{C}\right)$, it is sufficient to prove the statement when the base $Z$ is a curve. 
Let us write the canonical bundle formula for $f$ :

$$
K_{X}+B+\frac{1}{r}(\varphi)=f^{*}\left(K_{Z}+B_{Z}+M_{Z}\right)
$$

Let $V$ be a nonsingular model of the normalization of $X$ in $\mathbb{C}(X)(\sqrt[r]{\varphi})$.

(i): Let us suppose that $R^{d} h_{*} \mathbb{C}_{V_{0}}$ has unipotent monodromies. We argue as in [2], Theorems 4.5 and 0.1 .

The divisor $M_{Z}$ is a direct summand in $h_{*} \omega_{V / Z}$ (see [2, Lemma 5.2]) and since $\operatorname{deg} M_{Z}=0$ by [11] it defines a local complex subsystem of the variation of Hodge structure $R^{d} h_{*} \mathbb{C}$. By Corollary 4.14 there exists $m$ such that $m M_{Z} \cong \mathcal{O}_{Z}$ where

$$
m=m(b)=\operatorname{lcm}\{k \mid \phi(k) \leq b\}
$$

with $\phi$ the Euler function and $b=h^{d}(E, \mathbb{C})$.

(ii): Unipotent reduction: Consider a finite base change as in Proposition 4.12

$$
\tau: Z^{\prime} \rightarrow Z
$$

such that $R^{d} h_{*}^{\prime} \mathbb{C}_{V_{0}^{\prime}}$ has unipotent monodromies, where $h^{\prime}: V^{\prime} \rightarrow Z^{\prime}$ is the induced morphism. We have $\tau=\tau_{k} \circ \ldots \tau_{1}$ where

$$
\tau: Z^{\prime}=Z_{k+1} \stackrel{\tau_{k}}{\rightarrow} Z_{k} \ldots Z_{2} \stackrel{\tau_{1}}{\rightarrow} Z_{1}=Z .
$$

The morphism $\tau_{j}$ is a cyclic covering defined by the relation

$$
A_{j}^{\otimes \delta_{j}} \sim H_{j}
$$

where $A_{j}$ is a very ample divisor on $Z_{j}$. We know by case (i) that $m(b) M_{Z^{\prime}} \sim \mathcal{O}_{Z^{\prime}}$. By Theorem $5.1 \mathrm{~m}(b) M_{Z_{k}}$ is a Cartier divisor. We have thus the following isomorphisms: $\mathbb{C} \cong H^{0}\left(Z_{k+1}, m(b) M_{Z_{k+1}}\right) \cong H^{0}\left(Z_{k+1}, \tau^{*} m(b) M_{Z_{k}}\right) \cong H^{0}\left(Z_{k}, m(b) M_{Z_{k}} \otimes \tau_{*} \mathcal{O}_{Z_{k+1}}\right)$.

The second isomorphism is by Proposition 2.14 and the third follows from the projection formula. From the general theory about cyclic covers we have the isomorphism

$$
\tau_{*} \mathcal{O}_{Z_{k+1}} \cong \bigoplus_{l=0}^{\delta_{k}-1} A_{k}^{-l}
$$

Then we obtain

$$
H^{0}\left(Z_{k+1}, m(b) M_{Z_{k+1}}\right) \cong \bigoplus_{l=0}^{\delta_{k}-1} H^{0}\left(Z_{k}, m(b) M_{Z_{k}} \otimes A_{k}^{-l}\right) .
$$

Since $M_{Z} \equiv 0$, the divisor $m(b) M_{Z_{k}} \otimes A_{k}^{-l}$ has negative degree on $Z_{k}$ for all $l<0$, thus

$$
\mathbb{C} \cong H^{0}\left(Z_{k+1}, m(b) M_{Z_{k+1}}\right) \cong H^{0}\left(Z_{k}, m(b) M_{Z_{k}}\right)
$$

and $m(b) M_{Z_{k}} \sim \mathcal{O}_{Z}$. We can conclude by induction on $k$. 
Remark 6.2. Note that the same proof as in point (ii) of the proof of Theorem 1.3 implies a statement on Effective non-vanishing (see also Remark 3.4). More precisely assume that $\operatorname{deg} M_{Z}>0$ and $\tau: Z^{\prime} \rightarrow Z$ is as in Proposition 4.12, Assume that $H^{0}\left(Z^{\prime}, m(b) M_{Z^{\prime}}\right) \neq 0$. Then the reasoning above implies that $H^{0}\left(Z, m(b) M_{Z}\right) \neq 0$.

6.2. LC-trivial fibrations with numerically trivial moduli part. In this section we prove Theorem 1.4. To do so, we prove that $M_{Z}$ is a subsystem of a variation of Hodge structure related to the variation of mixed Hodge structure on $R^{d} \mathbb{C}_{V_{0} \backslash P_{V} / Z_{0}}$. We start with the following two results.

Proposition 6.3. Assume that for any lc-trivial fibration $f:(X, B) \rightarrow Z$ with

- $\operatorname{dim} Z=1$

- $M_{Z} \equiv 0$;

there exists an integer number $m$ such that $m M_{Z} \sim \mathcal{O}_{Z}$.

Then the same holds for bases $Z$ of arbitrary dimension.

Proof. The proof follows the same lines as the proof of Proposition 6.1.

Lemma 6.4 (Lemma 21, [14]). Let $\mathcal{L}$ be an invertible sheaf over a non-singular projective curve $C$, let $C_{0}$ be an open subset of $C$ and let $h$ be a metric on $\left.\mathcal{L}\right|_{C_{0}}$. Let $p$ be a point of $C \backslash C_{0}$ and let $t$ be a local parameter of $C$ centered at $p$. We assume that for a uniformizing section $v$ of $\mathcal{L}$ we have $h(v, v)=O\left(t^{-2 \alpha_{p}}|\log t|^{\beta_{p}}\right)$ for some real numbers $\alpha_{p}, \beta_{p}$. Then

$$
\operatorname{deg}_{C} \mathcal{L}=\frac{i}{2 \pi} \int_{C_{0}} \Theta+\sum_{p \in C \backslash C_{0}} \alpha_{p}
$$

where $\Theta$ is the curvature associated to $h$.

The following is a generalization of [3, Prop 3.4]

Proposition 6.5. Let $h: V \rightarrow Z$ be a fibration and let $\Sigma_{Z}$ be a simple normal crossing divisor such that

- $h$ is smooth over $Z_{0}=Z \backslash \Sigma_{Z}$,

- $\mathcal{W}_{l} / \mathcal{W}_{l-1}$ has unipotent monodromies, where $\left\{\mathcal{W}_{k}\right\}$ is the weight filtration.

Let $\mathcal{L}$ be an invertible sheaf such that $\left.\mathcal{L}\right|_{Z_{0}}$ is a direct summand of $\mathcal{W}_{l} / \mathcal{W}_{l-1}$ for some $l$. Assume that $\mathcal{L} \equiv 0$. Then $\left.\mathcal{L}\right|_{Z_{0}}$ is a local subsystem of $\mathcal{W}_{l} / \mathcal{W}_{l-1}$.

Proof. Since $\mathcal{W}_{l} / \mathcal{W}_{l-1}$ is a variation of Hodge structure, there is on it a flat bilinear form $Q$ and thus a metric and a metric connection. Then $\left.\mathcal{L}\right|_{Z_{0}}$ has an induced hermitian metric $h$, a metric connection and a curvature $\Theta$. To prove that $\left.\mathcal{L}\right|_{Z_{0}}$ defines a flat subsystem of $\mathcal{W}_{l} / \mathcal{W}_{l-1}$ it is sufficient to prove that the induced metric connection is flat, i.e. that $\Theta=0$.

The relation between the matrix $\Gamma$ of the connection and the matrix $H$ that represents the metric is $\Gamma=\bar{H}^{-1} \partial \bar{H}$. By Remark 4.9, the order near $p$ of the elements of $\Gamma$ is $O\left(|t|^{-1}|\log t|^{\beta_{p}}\right)$. Let $v$ be a uniformizing section of $\mathcal{L}$. Then the order of $h(v, v)$ near $p$ is $O\left(|\log t|^{\beta_{p}}\right)$. Let 
$C \subseteq Z$ be a curve such that $C \cap Z_{0} \neq \emptyset$. Let $\nu: \hat{C} \rightarrow C$ be its normalization and $C_{0}=C \cap Z_{0}$. We apply Lemma 6.4 and we obtain

$$
\operatorname{deg}_{C} \mathcal{L}=\frac{i}{2 \pi} \int_{\nu^{-1} C_{0}} \nu^{*} \Theta
$$

Since $\mathcal{L}$ is numerically zero, we obtain

$$
\int_{\nu^{-1} C_{0}} \nu^{*} \Theta=0
$$

for every $C$ and therefore $\Theta=0$.

Let us recall that we are working with a cyclic covering $V \rightarrow X$ of degree $r$, whose Galois group is the group of $r$-th roots of unity that we denote $\mu_{r}$. Moreover we have an induced action of $\mu_{r}$ on the sheaves of relative differentials. Let $P_{V}$ be the divisor given by the sum of the horizontal places of the pair $\left(V, B_{V}\right)$.

Lemma 6.6. The action of $\mu_{r}$ on $\left(h_{0}\right)_{*} \omega_{V_{0} / Z_{0}}\left(P_{V}\right)$ preserves the weight filtration

$$
\left\{\mathcal{W}_{k}\left(\left(h_{0}\right)_{*} \omega_{V_{0} / Z_{0}}\left(P_{V}\right)\right)\right\} \text {. }
$$

Proof. A generator of $\mu_{r}$ determines a birational map $\sigma: V \rightarrow V$. Consider a resolution of $\sigma$

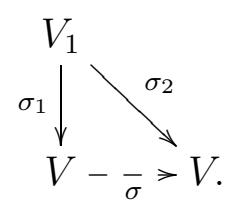

Let us consider the weight filtrations on $\Omega_{V_{0} / Z_{0}}^{\bullet}\left(\log P_{V}\right)$ and on $\Omega_{V_{10} / Z_{0}}^{\bullet}\left(\log P_{V_{1}}\right)$ where

$$
P_{V_{1}}=\operatorname{Supp}\left(\sigma_{2}^{-1} P_{V}\right) \text {. }
$$

The morphism $\sigma_{2}$ induces, for all $m$, the following:

$$
\sigma_{2}^{*}: \Omega_{V_{0} / Z_{0}}^{m}\left(\log P_{V}\right) \rightarrow \Omega_{V_{10} / Z_{0}}^{m}\left(\log P_{V_{1}}\right) .
$$

We want to prove that $\sigma_{2}$ preserves the weight filtration. Since $\sigma_{2}$ is a composition of blow-ups of smooth centres it is sufficient to prove the property for one blow-up.

Let $z_{1}, \ldots, z_{n}$ be a system of coordinates on $U \subseteq V_{0}$ such that

$$
P_{V} \cap U=\left\{z_{1} \cdot \ldots \cdot z_{k}=0\right\} .
$$

Then

$\sigma_{2}^{*}\left(\frac{d z_{1}}{z_{1}} \wedge \ldots \wedge \frac{d z_{h}}{z_{h}} \wedge d z_{h+1} \ldots \wedge d z_{n}\right)=\sigma_{2}^{*}\left(\frac{d z_{1}}{z_{1}}\right) \wedge \ldots \wedge \sigma_{2}^{*}\left(\frac{d z_{h}}{z_{h}}\right) \wedge \sigma_{2}^{*}\left(d z_{h+1}\right) \ldots \wedge \sigma_{2}^{*}\left(d z_{n}\right)$.

Let $C$ be the centre of the blow-up, let $z_{i}$ be one of the coordinates. There are two cases

(i): locally $C$ is contained in the zero locus of $z_{i}$;

(ii): $C$ is not contained in the zero locus of $z_{i}$. 
In case (i), let $t$ be an equation of the exceptional divisor and let $z_{i}^{\prime}$ be an equation of the strict transform of $z_{i}$. Then $\sigma_{2}^{*}\left(d z_{i}\right)=d\left(z_{i}^{\prime} \cdot t\right)=t \cdot d z_{i}^{\prime}+z_{i}^{\prime} \cdot d t$ and

$$
\sigma_{2}^{*}\left(\frac{d z_{i}}{z_{i}}\right)=\frac{t \cdot d z_{i}^{\prime}+z_{i}^{\prime} \cdot d t}{z_{i}^{\prime} t}=\frac{d z_{i}^{\prime}}{z_{i}^{\prime}}+\frac{d t}{t} .
$$

In case (ii), we simply have

$$
\sigma_{2}^{*}\left(d z_{i}\right)=d z_{i}^{\prime} \text { and } \sigma_{2}^{*} \frac{d z_{i}}{z_{i}}=\frac{d z_{i}^{\prime}}{z_{i}^{\prime}}
$$

Finally, the morphism $\sigma_{1}$ acts by pushforward and that does not increase the number of poles.

Proof of Theorem 1.4. By Proposition 6.3 we can assume that the base $Z$ is a curve.

Let us suppose that $\mathcal{W}_{k} / \mathcal{W}_{k-1}$ has unipotent monodromies for every $k$. Since by Lemma 6.6 the action of $\mu_{r}$ preservs the weight filtration

$$
\left\{\mathcal{W}_{k}\left(\left(h_{0}\right)_{*} \omega_{V_{0} / Z_{0}}\left(P_{V}\right)\right)\right\}
$$

on $V$, then for all $k$ the sheaf

$$
\mathcal{W}_{k}\left(\left(h_{0}\right)_{*} \omega_{V_{0} / Z_{0}}\left(\log P_{V}\right)\right)=h_{*}\left(\Omega_{V_{0} / Z_{0}}^{k}\left(\log P_{V}\right) \otimes \Omega_{V_{0} / Z_{0}}^{n-k}\right)
$$

decomposes as sum of eigensheaves. In particular, since $\mathcal{O}_{Z}\left(M_{Z}\right)$ is an eigensheaf of rank one of $h_{*} \omega_{V / Z}\left(P_{V}\right)$, there exist $l$ such that $\left.\mathcal{O}_{Z}\left(M_{Z}\right)\right|_{Z_{0}} \subseteq W_{l}$ and $\left.\mathcal{O}_{Z}\left(M_{Z}\right)\right|_{Z_{0}} \not \mathcal{W}_{l-1}$. Thus there exists $\mathcal{V}$ containing $\mathcal{W}_{l-1}$ such that $\mathcal{W}_{l}=\left.\mathcal{O}_{Z}\left(M_{Z}\right)\right|_{Z_{0}} \oplus \mathcal{V}$. By Proposition 6.5 thus $\left.\mathcal{O}_{Z}\left(M_{Z}\right)\right|_{Z_{0}}$ defines a local subsystem of $\mathcal{W}_{l} / \mathcal{W}_{l-1}$. By Corollary 4.14 we have $\left.\mathcal{O}_{Z}\left(M_{Z}\right)\right|_{Z_{0}} ^{m(h)} \sim \mathcal{O}_{Z_{0}}$ with $h=\mathrm{rk} \mathcal{W}_{l} / \mathcal{W}_{l-1}$. Since if the monodromies are unipotent the canonical extension commutes with tensor product, we have $\mathcal{O}_{Z}\left(M_{Z}\right)^{m(h)} \sim \mathcal{O}_{Z}$.

The general situation, when $\mathcal{W}_{k} / \mathcal{W}_{k-1}$ has not unipotent monodromies for every $k$, can be reduced to the unipotent situation. We take a covering $\tau: Z^{\prime} \rightarrow Z$ such that on $Z^{\prime}$ we have unipotent monodromies. Then $m(h) M_{Z^{\prime}} \sim \mathcal{O}_{Z^{\prime}}$ and since $M_{Z^{\prime}}=\tau^{*} M_{Z}$ we have $\operatorname{deg} \tau \cdot m(h) M_{Z} \sim \mathcal{O}_{Z}$.

\section{REFERENCES}

[1] F. Ambro, The Adjunction Conjecture and its applications, PhD thesis, The Johns Hopkins University, arXiv:math/9903060.

[2] F. Ambro, Shokurov's boundary property, J. Differential Geom. 67 (2004), no. 2, 229-255.

[3] F. Ambro, The moduli b-divisor of an lc-trivial fibration, Compos. Math. 141 (2005), no. 2, 385-403.

[4] A. Corti, Flips for 3-folds and 4-folds, Oxford Lecture Ser. Math. Appl. 35, Oxford Univ. Press, Oxford (2007).

[5] P. Deligne, Équations différentielles à Points Singuliers Réguliers, Lecture Notes in Mathematics 163, Springer-Verlag, Berlin-New York, (1970).

[6] P. Deligne, Théorie de Hodge II, Inst. Hautes Études Sci. Publ. Math. No. 40 (1971) 5-57.

[7] E. Floris, Bounds on the denominators in the canonical bundle formula arXiv:1105.4553.v3 to appear in Ann. Inst. Fourier (Grenoble). 
[8] O. Fujino, A canonical bundle formula for certain algebraic fiber spaces and its applications, Nagoya Math. $J 172$ (2003), 129-171.

[9] O. Fujino, Algebraic fibre space whose general fibers are maximal albanese dimension, Nagoya Math. $J$ 172 (2003), 111-127.

[10] O. Fujino, S. Mori, A canonical bundle formula, J. Differential Geom. 56 (2000), no. 1, 167-188.

[11] T. Fujita, The sheaf of relative canonical forms of a Kähler fiber space over a curve, Proc. Japan Acad. Ser. A Math. Sci. 54 (1978), no. 7, 183-184.

[12] X. Jiang, On the pluricanonical maps of varieties of intermediate Kodaira dimension, arXiv:1012.3817.v2.

[13] N. Katz, Nilpotent connections and the monodromy theorem. Applications of a result of Turrittin. Inst. Hautes Études Sci. Publ. Math. No. 39 (1971), 175-232.

[14] Y. Kawamata, Characterization of abelian varieties, Compositio Math. 43 (1981), no. 2, 253-276.

[15] Y. Kawamata, Kodaira dimension of certain algebraic fiber spaces, J. Fac. Sci. Univ. Tokyo Sect. IA Math. 30 (1983), no. 1, 1-24.

[16] J. Kollár, Higher Direct Images of Dualizing Sheaves II, Ann. of Math. (2) 124 (1986), no. 1, 171-202.

[17] J. Kollár, S. Mori, Birational Geometry of Algebraic Varieties, Cambridge Tracts in Mathematics 134, Cambridge University Press, Cambridge (1998).

[18] S. Mori, Classification of Higher-Dimensional Varieties, Proc. Sympos. Pure Math., 46 (1987).

[19] C. Peters, J. Steenbrink, Mixed Hodge structures, Ergebnisse der Mathematik und ihrer Grenzgebiete. 3. Folge. A Series of Modern Surveys in Mathematics [Results in Mathematics and Related Areas. 3rd Series. A Series of Modern Surveys in Mathematics], 52. Springer-Verlag, Berlin, (2008).

[20] Y. Prokhorov, V. Shokurov, Towards the second main theorem on complements J. Algebraic Geom. 18 (2009), no. 1, 151-199.

[21] J. Steenbrink, S. Zucker, Variation of mixed Hodge structure I, Invent. Math. 80 (1985), no. 3, 489-542.

$[22]$ G. T. Todorov, Effective log Iitaka fibrations for surfaces and threefolds, Manuscripta Math. 133 (2010), no. 1-2, 183-195.

[23] G. T. Todorov, C. Xu, Effectiveness of the log Iitaka fibration for 3-folds and 4-folds, Algebra Number Theory 3 (2009), no. 6, 697-710.

Enrica Floris, IRMA, Université de Strasbourg et CNRS, 7 rue René-Descartes, 67084 Strasbourg Cedex, France

E-mail address: floris@math.unistra.fr 